\title{
ON THE ESTIMATION OF GLYCERINE IN FATS.
}

\section{By Otтo Hehner.}

Read at the Meeting, February 9th, 1887.

IN the publication of the principle upon which the method to be described in the following paper is based, I have quite recently been forestalled by L. Legler (ANALYsT, January 1887), and I, therefore, cannot lay any claim to originality. But as I operate in a manner quite different from that adopted by Legler-his process being only applicable to somewhat concentrated glycerine liquor, whilst I am enabled to determine the glycerine in even the most dilute solutions-I venture to lay a description of it, and of results obtained, before the members of the Society. A portion of my investigation is, furthermore, of general importance, and concerns all methods of glycerine estimation, since it treats of the question of the volatility of glycerine with aqueous vapour.

Glycerine decomposes, on treatment with bichromate of potassium and sulphuric acid, into carbonic acid and water. Legler weighs the carbonic acid, or rather the loss of carbonic acid, in an ordinary carbonic acid apparatus. Messrs. Cross and Bevan (Chemical News, Vol. 56, p. 2) measure the volume of the gas evolved.

It is evident that both these modifications require limited bulks of fluid, and, therefore, exclude the estimation of glycerine in very dilute solutions, such as are obtained in the analysis of fat-the washings, in fact, of the insoluble fatty acids.

The process which I have described (ANALYst, XII, February) for the estimation of methyl in the presence of ethylalcohol, and which consists in the measurement of the quantity of bichromate reduced, is, as I hope to show, particularly suitable for the analysis of such washings.

One part of glycerine requires, for complete oxydation, $7 \cdot 486$ parts of potassium bichromate.

Solutions required.-(1.) Bichromate, containing in each litre about 80 grammes of bichromate and 150 c.c. of strong sulphuric acid. The exact value of the solution should be ascertained by titration with solutions of known weights of iron wire.

2. Ferrous and ammonia sulphate containing about 120 grms. per litre.

3. Bichromate ten times more dilute than the above.

The ferrous solution is exactly standardised upon the chromate solution, and the glycerine value of the chromate (contents of $\mathrm{K}_{2} \mathrm{C}_{2} \mathrm{O}_{7}$ divided by $7 \cdot 486$ ) is calculated.

The chromate solution used in my experiments standardised as follows :-

2.8412 grms. iron-wire $=2.8327$ pure iron, required 33.94 c.c. bichrome. 1 c.c. $=$ $\cdot 07333$ bichrome, or 009796 glycerine.

$2 \cdot 7078$ grms. iron-wire $=2 \cdot 6997$ iron used $32 \cdot 3$ c.c., bichrome 1 c.c. $=07344$ 
bichrome or $\cdot 009810$ glycerine. Average 1 c.c. bichrome $=\cdot 07338$ grms. bichrome or .009803 glycerine.

Test Experiments.-Glycerine, specific gravity 1.2572, containing, according to Lenz's tables, $95 \cdot 55$ per cent. of pure anhydrous glycerine-was taken, a solution of $12 \cdot 5798$ grme. per litre, corresponding to 12.0200 grms. of pure glycerine, being used. 25 c.c. of this solution, equal to 3005 glycerine were taken in each of the following experiments.

25 c.c heated with 40 c.c. bichromate, without further dilution for two hours to near the boiling point: $30 \cdot 41$ c.c. bichromate were consumed, corresponding to $\cdot 2981 \mathrm{grms}$. glycerine, or 99.2 per cent. of the glycerine taken.

25 c.c., heated with bichrome and 25 c.c. strong hydrochloric acid for one hour, consumed $31 \cdot 16$ c.c. bichrome, equal to 30546 grms. or $101 \cdot 6$ per cent.

25 c.c., diluted with 500 c.c. of distilled water, reduced in three hours 23.93 c.c. bichromate, equal to 23458 grm. or $78 \cdot 1$ per cent of glycerine.

The same quantity, diluted with 500 c.c. of water, plus 25 c.c. strong hydrochoric acid, heated for one hour, reduced $22 \cdot 3$ c.c. bichrome, indicating $\cdot 2186$ grms., or $72 \cdot 7$ per cent.

25 c.c., diluted with 500 c.c. of water, plus 25 c.c. sulphuric acid, consumed in one hour 29.34 c.c. bichrome, $=\cdot 2876$ grms., or 95.7 per cent. of the glycerine taken.

Conditions the same as in the previous experiment, only the heating continued for two hours. Bichromate consumed 29.89 c.c. $==2930$ grms, or 97.5 per cent. of glycerine taken.

25 c.c. were diluted to about 300 c.c., the solution evaporated to about one-half upon the water bath, and then heated with 25 c.c. strong sulphuric acid and chromate. After two hours 30.54 c.c. bichromate were found to be reduced, corresponding to $\cdot 2994$ grms. or 99.6 per cent. of glycerine.

In a similar experiment the diluted solution was vigorously boiled down over the naked flame to about one half (the basin being, of course, covered with a dock glass to prevent loss by spurting), and heated for two hours with sulphuric acid and bichromate. Found $\cdot 2961$ grms. or $98 \cdot 5$ per cent. of the glycerine taken.

In a precisely analogous experiment 10 c.c. of strong alcohol were added to the water, before boiling, over the naked flame. After two hours 30.33 c.c. of chromate were reduced, equal to $\cdot 2973$ grms. or 98.9 per cent. of glycerine found.

The alcohol experiment repeated, but the fluid concentrated on the water bath, an amount of bichromate was reduced corresponding to 107.6 per cent. of glycerine. An odour of aldehyde was perceptible during the oxidation.

Deductions.-From these test experiments the following conclusions can be drawn.

1. In a fairly concentrated solution glycerine is quantitatively oxidised by acid bichromate.

2. In a very dilute solution the oxidation is not complete even after many hours' heating.

3. The addition of hydrochloric acid does not materially help oxidation.

4. In solutions containing about 10 per cent. of strong sulphuric acid the oxidation is complete after two hours, even in exceedingly dilute solutions ( 6 glycerine per 1000 of fluid).

5. From such dilute solutions, glycerine does not, as is commonly assumed, volatilise on concentrating the fluid, be it on the water bath or over the naked fame. 
6. Should alcohol be present it is completely volatilised by vigorously boiling the fluid d swn to one half, but not on the water bath.

The non-volatility of glycerine from dilute solutions may further be readily demonstrated by distilling from a large retort 500 c.c. of water, containing about $\cdot 3$ grms. of glycerine, catching the first 250 c.c. of the distillate. This distillate does not, even on heating, decolourise more than'a few drops of a dilute permanganate solution, such as is used in water analysis.

Method for Estimating Glycerine in Fats.-Saponify about 3 grms. of the fat with alcoholic potash; do not drive off all the alcohol, lest glycerine should volatilise from the concentrated solution, but dilute to about 200 c.c. ; decompose the soap with dilute sulphuric acid, filter off, and estimate insoluble fatty acids as usual. Vigorously boil the filtrate and washings (amounting to about 500 c.c.) in a covered beaker or basin, down to one-half, add 25 c.c. strong sulphuric acid (suitably diluted) and 50 c.c. standard bichromate. Heat to near boiling for two hours, and titrate back the excess of bichromate with excess of ferrous sulphate, and ultimately the latter with decichromate using ferricyanide as indicator. Calculate from the chromate consumed the amount and percentage of glycerine.

Finally, I will add a few results obtained on applying the method to a few fats :

$\begin{array}{llllll}\text { Olive oil } & \ldots & \ldots & \ldots & \ldots & 10 \cdot 26 \text { per cent. glycerine. } \\ \text { Cod liver oil } & \ldots & \ldots & \ldots & \ldots & 9 \cdot 87 \text { per cent. } \\ \text { Linseed oil } & \ldots & \ldots & \ldots & \ldots & 10 \cdot 24 \text { and } 10.20 \text { per cent. } \\ \text { Butterine } & \ldots & \ldots & \ldots & \ldots & 10.01 \text { per cent. } \\ \text { Butter } & \ldots & \ldots & \ldots & \ldots & 12.40 \text { and } 11.96 \text { per cent. }\end{array}$

Of course, I am fully aware that other substances, should they be present, might reduce bichromate as well as the glycerine does. The analgous objection applies to Dr. Muter's and Mr. Fox's processes; I have, however, convinced myself that soluble fatty acids, like butyric, do not act upon bichromate, nor do fatty acids of higher molecular value.

The method may not be theoretically perfect, but it may commend itself for its simplicity and rapidity in cases of fluids which cannot contain anything but glycerine and soluble fatty acids.

Note.-I here correct two errors that have crept into my paper on methylalcohol.

Dr. Dupré did find 58 , not 42 per cent. of methylalcohol, for 10 per cent. of methylated spirit.

The ferrous sulphate solution employed contains 120, not 240, grammes of iron and ammonia sulphate per litre. 